\title{
EL MOVIMIENTO DE UNIVERSIDADES PROMOTORAS DE LA SALUD
}

\author{
Hiram V. Arroyo \\ Escuela de Salud Pública - Universidad de Puerto Rico - San Juan - Puerto Rico \\ Centro Colaborador de la Organización Panamericana de la Salud - Organización Mundial de la Salud - OMS/OPS - San Juan - Puerto Rico \\ Red Iberoamericana de Universidades Promotoras de la Salud (RIUPS) - San Juan - Puerto Rico
}

Las Universidades Promotoras de la Salud (UPS) tienen la responsabilidad de generar una cultura de Promoción de la Salud en el campus y en el ambiente social y comunitario externo. Las UPS se comprometen a impulsar el marco operacional de la Promoción de la Salud contenido en la Carta de Ottawa y ratificado en documentos declaratorios de salud posteriores a nivel internacional. También las UPS tienen la misión de la abogacía en salud utilizando como marco conceptual/operacional los postulados de los Determinantes Sociales de la Salud (DSS) y los Objetivos del Desarrollo Sostenible (ODS) $)^{(1-3)}$.

En el Cuadro I se presenta un resumen de los componentes de la definición de UPS:

El Movimiento de Universidades Promotoras de la Salud (UPS) continua su ruta ascendente en América Latina. La iniciativa de UPS se activa a nivel regional a partir del 2003. Han sido años de grandes avances políticos y programáticos para la Promoción de la Salud en el entorno universitario. Algunos de los avances se presentan a continuación ${ }^{(4,5)}$ :

a) Quince años del Movimiento de Universidades Promotoras de la Salud (UPS) 2003-2018. b) Ocho Congresos Internacionales de Universidades Promotoras de la Salud (UPS) efectuados en Santiago de Chile (2003); Edmonton, Canadá (2005); Ciudad Juárez, México (2007); Pamplona, España (2009); San José de Costa Rica (2011); San Juan de Puerto Rico (2013); Kelowna, British Columbia (2015), Canadá, y Alicante, España (2017). c) Siete documentos declaratorios (Edmonton, Ciudad Juárez, Pamplona, San José, San Juan, Okanagan, Alicante). d) Incremento de instituciones universitarias afiliadas. e) Surgimiento de once estructuras de Redes Nacionales de UPS en: Brasil, Chile, Centroamérica y El Caribe, Colombia, Costa Rica, Cuba, Ecuador, España, México, Perú, y Puerto Rico. f) Surgimiento de Redes Estatales y otras modalidades de trabajo interinstitucional. g) Aumento de publicaciones, eventos y reuniones técnicas sobre el tema.

La iniciativa de UPS compromete a las universidades y a otras entidades de Educación Superior a lo siguiente:

Generar el compromiso político institucional para ubicar a la Promoción de la Salud como elemento integrador de la visión, misión, valores y el plan estratégico universitário; Operacionalizar el enfoque de Universidad Promotora de la Salud (UPS) al interior de la institución a través de una estructura y programa formal de actividades sistemáticas de Promoción de la Salud donde participen docentes, estudiantes y demás miembros de la comunidad universitária; Implica la designación de funcionarios universitarios responsables de coordinar el programa de UPS y garantizar la sistematización de acciones con el apoyo de representantes de diferentes Facultades, Escuelas y disciplinas de estúdio; Rendir informes periodicos de las acciones de Promoción de la Salud que emprende la Universidad a nivel interno en el campus y a nivel externo con su vinculación directa en los asuntos sociales, asuntos de políticas públicas favorables a la salud, acciones intersectoriales y de abogacía en salud ${ }^{(6)}$.

Ha habido una experiencia expansionista de las redes de Universidades Promotoras de la Salud en la Región Iberoamericana. El desarrollo de las redes nacionales ha sido un ámbito de trabajo impulsado por la Red lberoamericana de Universidades Promotoras de la Salud (RIUPS). En el Cuadro II se presenta la relación de las redes existentes al presente. Las redes nacionales de Universidades Promotoras de la Salud están ejerciendo un rol significativo 
en los avances del Movimiento de Promoción de la Salud y la consolidación del enfoque salubrista de entornos saludables ${ }^{(6-8)}$. Las redes nacionales son responsables de los siguientes avances:

Visibilizar los valores de la Promoción de la Salud a nivel nacional y en las instituciones universitarias; Difundir las acciones políticas y de desarrollo de capacidad en Promoción de la Salud a nivel regional y global. Esto incluye la divulgación de eventos y conferencias nacionales e internacionales en el tema de Promoción de la Salud; Mantener una relación de intercambio técnico y científico en el ámbito de la Promoción de la Salud con la Organización Panamericana de la Salud y los Centros Colaboradores de la OMS/OPS de la región; Incidir en las políticas de salud a nivel nacional a través de las estructuras de Promoción de la Salud de los Ministerios de Salud; Defender la unicidad del proyecto nacional de Universidad Promotora de la Salud respetando las características culturales particulares de cada país; Desarrollar capacidad institucional en el ámbito de la Promoción de la Salud y la Educación para la Salud; Comparar avances conceptuales y metodológicos con otras redes nacionales lo que implica el desarrollo de capacidades a nivel de la salud global; Ampliar las acciones de intercambio académico-profesional con otras universidades en la esfera nacional e internacional; Participar en comisiones de trabajo interpaíses con la finalidad de desarrollar proyectos y acciones programáticas de interés común entre las instituciones representadas; Ampliar la vinculación con otras entidades profesionales de la región incluyendo la Oficina Regional Latinoamericana (ORLA) de la Unión Internacional de Promoción de la Salud y Educación para la Salud (UIPES) ${ }^{(6,7)}$.

Las funciones de la Red Iberoamericana de Universidades Promotoras de la Salud (RIUPS) son: Participar en la organización de las Reuniones Internacionales bianualmente; Promover el reclutamiento y la adhesión de nuevas UPS como miembros de la RIUPS; Mantener la documentación de las Reuniones y actividades de las UPS; Vigilar el cumplimiento de los criterios para ser una UPS; Proveer apoyo para el monitoreo y evaluación de las experiencias de las UPS; Diseminar las experiencias y lecciones aprendidas con las UPS.

La Red de Brasil de Universidades Promotoras de la Salud se oficializa en el 2018 por iniciativa de la Universidad de Brasilia y otras Universidades que apoyaron su creación. A continuación se presentan algunas de las Universidades que se han integrado a la Red: Universidad de Fortaleza, Universidad de Franca, Universidad de Brasília, Universidad Federal do Rio Grande do Sul, Centro Universitario de Maringá, Universidad Estadual de Goiás, Universidad Federal de Minas Gerais, Universidad Federal de Santa Catarina, Universidad Federal de Paraíba, Universidad Federal de Mato Grosso do Sul, Centro Universitario Euro-Americano, Universidad Federal da Integração Latino-Americana, Universidad do Estado do Amazonas, Universidad Metodista de Sao Paulo, Universidad Federal do Espirito Santo, Universidad Federal de Mato Grosso, Universidad Federal de Goiás y Universidad Federal de Ciencias de la Salud de Porto Alegre, Brasil.

Lograr mayor visibilidad de las UPS en las estructuras e instancias de toma de decisiones en el ámbito de la Educación Superior; Expandir la difusión del discurso y la evidencia de las prácticas efectivas del Movimiento de Universidades Promotoras de la Salud a nivel nacional e internacional; Mantener las acciones de liderazgo activo de las Redes nacionales de Universidades Promotoras de la Salud; Promover el desarrollo de las Comisiones de Trabajo de la RIUPS y el intercambio de saberes y experiencias entre las universidades participantes ${ }^{(9)}$.

Así, esperamos con la edición especial de la Revista Brasileña de Promoción de la Salud contribuir para la discusión acerca de las universidades promotoras de salud.

\section{Cuadro I - Componentes de la definición de Universidades Promotoras de la Salud (UPS)*.}

\begin{tabular}{|c|c|c|}
\hline Ejes transversales de las UPS & Ámbitos de actuación & Definiciones operacionales \\
\hline $\begin{array}{l}\text { Perspectiva de los Determinantes } \\
\text { sociales de la salud (DSS). } \\
\text { Perspectiva de los Objetivos de } \\
\text { Desarrollo Sostenible (ODS). }\end{array}$ & $\begin{array}{l}\text { Desarrollo de normativa y } \\
\text { políticas institucionales para } \\
\text { propiciar una cultura universitaria } \\
\text { y un ambiente favorable a la } \\
\text { salud. }\end{array}$ & $\begin{array}{l}\text { - Impulsar acciones de abogacía en salud. } \\
\text { - Participar en procesos de análisis de política pública } \\
\text { en salud. } \\
\text { - Utilizar enfoques de trabajo intersectorial y multidisciplinario. }\end{array}$ \\
\hline \multirow[t]{2}{*}{$\begin{array}{l}\text { Alcance social, familiar y } \\
\text { comunitario. }\end{array}$} & $\begin{array}{l}\text { Desarrollo de acciones de } \\
\text { Educación para la salud. }\end{array}$ & $\begin{array}{l}\text { - Incorporar las perspectivas de trabajo asociadas a la } \\
\text { alfabetización en salud y la comunicación en salud. } \\
\text { - Fomento de las habilidades para la vida. } \\
\text { - Fomento del liderato estudiantil en salud. }\end{array}$ \\
\hline & $\begin{array}{l}\text { Ofrecimiento de servicios } \\
\text { sociales, servicios médicos y } \\
\text { de salud integral. }\end{array}$ & $\begin{array}{l}\text { - Combinación de servicios médicos, educación para la } \\
\text { salud, psicológicos y de trabajo social. } \\
\text { - Visión amplia de servicios que cobije los determinantes } \\
\text { sociales. }\end{array}$ \\
\hline
\end{tabular}




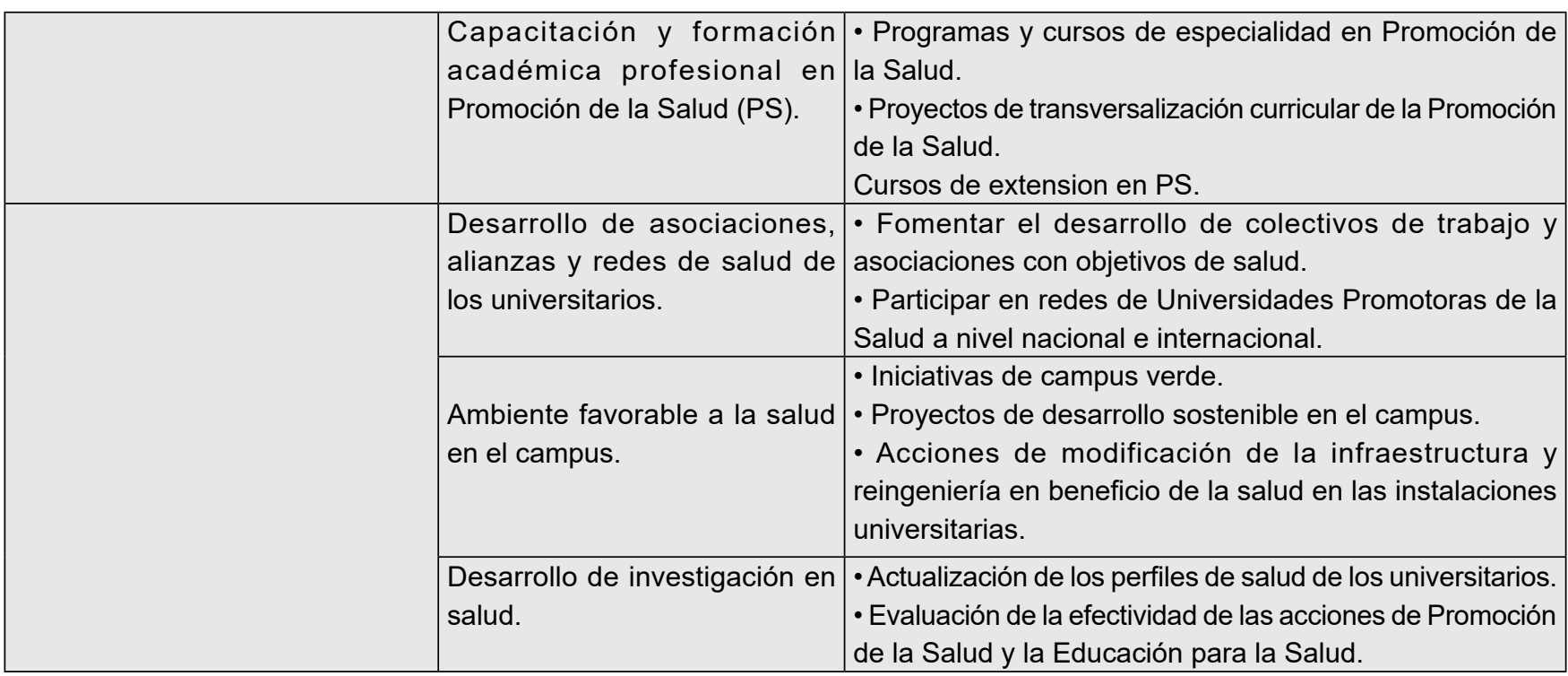

* Red Iberoamericana de Universidades Promotoras de la Salud (RIUPS, 2013). Definición adoptada por la RIUPS en su Asamblea General efectuada en San Juan de Puerto Rico.

Cuadro II - Relación de las RIUPS (Red Iberoamericana de Universidades Promotoras de la Salud) existentes.

\begin{tabular}{|l|l|l|}
\hline Redes Iberoamericanas activas & $\begin{array}{l}\text { Redes en proceso de desarrollo } \\
\text { en Iberoamérica }\end{array}$ & Otras redes globales \\
\hline Brasil & Argentina & Alemania \\
Colombia & Guatemala & Canadá \\
Costa Rica & Honduras \\
Chile & Nicaragua & Inglaterra \\
Cuba & Panamá \\
Ecuador & Portugal & \\
España & Uruguaya & \\
México & & \\
Perú & & \\
Puerto Rico & & \\
Red Centroamericana y del Caribe de UPS & & \\
\hline
\end{tabular}

\section{REFERENCIAS}

1. Arroyo HV. Valoración del movimiento de Universidades Promotoras de la Salud y la Red Iberoamericana de Universidades Promotoras de la Salud (RIUPS) [internet]. 2018; [acesso em: 2018 fev.2]. Publicación en proceso para el libro de la Red Mexicana de Universidades Promotoras de la salud. Acesso em: 2 fev 2018. Disponível em: https://www.paho.org/hq/index.php?option=com_content\&view=article\&id=10675:2015-iberoamericannetwork-of-health-promoting-universities-riups\&ltemid=820\&lang=es

2. Arroyo HV. Las Universidades Promotoras de la Salud: conceptuación y relevancia global. In: Chávez, Aída Imelda Valero, editor. Promoción, alfabetización e intervención en salud: experiencias innovadoras desde la multidisciplina. Cidade do México: Universidad Nacional Autónoma de México (UNAM) En proceso de publicación; 2018.

3. Arroyo HV. Editorial Mirada al Movimiento Iberoamericano de Universidades Promotoras de la Salud. Horizonte Sanitario. 2017;16(3):149-51.

4. Arroyo HV, Durán G, Gallardo C. Diez años del Movimiento de Universidades Promotoras de la Salud en Iberoamérica y la contribución de la Red Iberoamericana de Universidades Promotoras de la Salud (RIUPS). Global Health Promotion. 2015;22(4):64-8. 
5. Arroyo HV. El Movimiento Iberoamericano de Universidades Promotoras de la Salud: conceptuación y práctica. San Juan: Editorial de la Universidad de Puerto Rico; 2013.

6. Declaración de Alicante sobre la Promoción de la Salud y Universidad Construyendo Entornos Sociales y Educativos Saludables. $18 .^{\circ}$ Congreso Iberoamericano de Universidades Promotoras de la Salud en Alicante; 2017 Jun 27 - 29; Alicante: Universidad de Alicante; 2017 Jun.

7. Declaración de Ciudad Juárez. Acta Constitutiva de la Red Iberoamericana de Universidades Promotoras de la Salud (RIUPS). $3 .^{\circ}$ Congreso Internacional de Universidades Promotoras de la Salud; 2007 Oct 3 - 6; Juárez: Universidad Autónoma de Ciudad Juárez; 2007 Oct.

8. Diez para la Década del Movimiento de Universidades Promotoras de la Salud. $6 .^{\circ}$ Congreso Iberoamericano de Universidades Promotoras de la Salud; 2013 Mar 19-21; San Juan de Puerto Rico: Universidade de Puerto Rico; 2013 Mar.

9. Martínez JR, Gallardo C, Aguiló A, Granados MC, López J, Arroyo HV. La universidad como comunidad: universidades promotoras de salud. Informe SESPAS. Gac Sanit. 2018;32(S1):86-91.

\section{Endereço para correspondência:}

Hiram V. Arroyo

Recinto de Ciencias Médicas - Escuela de Salud Publica

Edificio Dr. Guillermo Arbona Irizarry, 4to piso

Oficina A - 403

Área Centro Médico

Río Piedras - Puerto Rico - 00935

E-mail: hiram.arroyo1@upr.edu 